\title{
Sobre um Problema de Transformação Conforme
}

L. BOURChtein, 1 A. BOURChtein, 2 A.N. OliveirA, ${ }^{3}$ Departamento de Matemática, Estatística e Computação, IFM, UFPel, 96010-900 Capão do Leão, RS, Brasil.

Resumo. Neste artigo é analisada a transformação conforme do quadrado unitário no círculo unitário de tal modo que os vértices do quadrado passam em quatro pontos eqüidistantes da circunferência unitária. Para construirmos esta transformação, utilizamos as funções elementares, os princípios básicos, o princípio de simetria de Riemann-Schwarz e, principalmente, a integral de Schwarz-Christoffel.

\section{Introdução}

O conceito de transformação conforme é um dos conceitos importantes da matemática. Este conceito surgiu da resolução de alguns problemas físicos e tem várias aplicações em diferentes ramos da física: hidrodinâmica, aeronáutica, teoria dos campos magnéticos, eletrostáticos e outros. Com o desenvolvimento dos métodos numéricos para a resolução de equações diferenciais parciais as transformações conformes começaram a ser utilizadas, também, na geração de grades computacionais. O problema fundamental da teoria das transformações conformes é o seguinte: dadas duas regiões, é preciso construir uma função que realiza transformação conforme de uma dessas regiões à outra. Nem sempre a transformação conforme é possível, existem algumas condições necessárias para essas transformações. Por exemplo, não existe tranformação conforme de uma região à outra se essas regiões têm diferentes graus de conexão [3]. Para regiões simplesmente conexas existe o teorema de Riemann que afirma que qualquer região simplesmente conexa, diferente de todo plano, pode ser transformada biunivocamente no círculo unitário [2,3]. Mas, realizar essa transformação de forma concreta é um problema complicado, pois não existe um único algoritmo para construção das transformações conformes.

Neste trabalho, analisaremos a transformação conforme do quadrado unitário no círculo unitário de tal modo que os vértices do quadrado passam em quatro pontos eqüidistantes da circunferência unitária. Esta transformação foi utilizada em [1] na investigação da validade da utilização das equações generalizadas de Laplace para geração de grades ortogonais computacionais. O último problema mencionado tem ligação íntima com a resolução numérica de problemas complexos de geometria [5]. Em [1] foram apenas utilizadas algumas propriedades desta transformação. No

\footnotetext{
${ }^{1}$ burstein@terra.com.br

2 burstein@ufpel.tche.br

3 adriana_neumann@terra.com.br
} 
trabalho apresentado consideraremos a construção completa dessa transformação. Para realizar a transformação conforme de um quadrado no círculo com a correspondência indicada dos vértices usaremos, além das funções elementares e os princípios básicos das tranformações conformes $[2,6]$, o princípio de simetria de Riemann-Schwarz [6, 7] e a integral de Schwarz-Christoffel $[2,6]$. Então, relembraremos alguns conceitos e resultados importantes para a construção feita neste trabalho.

Definição 1.1. Uma função $w=f(z)$ é chamada regular num ponto $z_{0}$, se $f(z)$ pode ser desenvolvida numa série de potências

$$
f(z)=\sum_{n=0}^{+\infty} c_{n}\left(z-z_{0}\right)^{n}
$$

que converge num círculo $\left|z-z_{0}\right|<r$ com centro no ponto $z_{0}$.

Definição 1.2. Uma função $w=f(z)$, definida na região $D$, é chamada regular nessa região se ela é regular em qualquer ponto dessa região.

É conhecido $[2,7]$ que a regularidade de uma função numa região é equivalente à diferenciabilidade dessa função na mesma região.

Definição 1.3. A função $f(z)$ é chamada univalente numa região $D$, se $\forall z_{1}, z_{2} \in D$, $z_{1} \neq z_{2}$, segue que $f\left(z_{1}\right) \neq f\left(z_{2}\right)$.

Se considerarmos $f(z)$ unívoca e univalente na região $D$, neste caso então teremos $f(z)$ biunívoca.

Definição 1.4. Se a função $f(z)$ é regular e univalente na região $D$, então $f(z)$ realiza transformação conforme dessa região.

Teorema 1.1 (Princípio da Simetria de Riemann-Schwarz, [7]). Seja D alguma região cuja fronteira contém o segmento $\gamma$ do eixo real. Seja a função $w=f(z)$ regular na região $D$ e contínua em $D \cup \gamma$ e $f(z)$ assume valores reais no segmento $\gamma$, isto é, a imagem de $\gamma$ é um segmento $\Gamma$ que está sobre o eixo real através da transformação $f(z): \Gamma=f(\gamma)$. Então, a função $f(z)$ pode ser prolongada analiticamente da região $D$ para a região $D^{\prime}$ que é simétrica à $D$ em relação ao eixo real. E o prolongamento dessa função define-se do seguinte modo:

$$
F(z)=\left\{\begin{array}{ll}
f(z), & z \in D \cup \gamma \\
\overline{f(\bar{z})}, & z \in D^{\prime}
\end{array} .\right.
$$

Observações:

1. Denotamos por $G$ a imagem da região $D$ e por $G^{\prime}$ a imagem da região $D^{\prime}$ sob a transformação $F(z)$. Da fórmula (1.1) é visto que não somente $D$ e $D^{\prime}$ são simétricos em relação ao eixo real, mas suas imagens $G$ e $G^{\prime}$ também são. 
2. Denotamos por $D^{*}$ a região do plano $z$, ou seja, $D^{*}=D \cup \gamma \cup D^{\prime}$ e por $G^{*}$ a região do plano $w$, assim, $G^{*}=G \cup \Gamma \cup G^{\prime}$. Se a região $D$ é totalmente contida no semiplano superior ou semiplano inferior, então neste caso as regiões $D$ e $D^{\prime}$ não intercecsionam-se: $D \bigcap D^{\prime}=\emptyset$. Assim, a região $D^{*}$ é univalente e a função $F(z)$, determinada pela fórmula (1.1), é função unívoca na região $D^{*}$.

3. Sabemos [6] que para uma função realizar transformação conforme ela precisa ser univalente. Assim a função $F(z)$ somente realizará transformação conforme se for biunívoca. Para que isso aconteça, além de $D^{*}$ ser como na observação anterior, ou seja, $D \bigcap D^{\prime}=\emptyset$, temos que ter também $G \bigcap G^{\prime}=\emptyset$, isto é, cada ponto de $G^{*}$ pertencerá a $G, G^{\prime}$ ou $\Gamma$.

Definição 1.5. A integral de Schwarz-Christoffel (integral elíptica de $1^{a}$ espécie) tem a seguinte forma

$$
w=F(z, k)=\int_{0}^{z} \frac{d t}{\sqrt{\left(1-t^{2}\right)\left(1-k^{2} t^{2}\right)}}, 0<k<1, \quad \text { Im } z>0 .
$$

O parâmetro $k$ chama-se módulo da integral (1.2) e o número $k^{\prime}, k^{2}+k^{\prime 2}=1$, é o módulo adicional.

A função (1.2) transforma biunivocamente o semiplano superior num retângulo de tal modo que os pontos do eixo real $1,1 / k,-1 / k,-1$ passam, respectivamente, nos pontos $K, K+i K^{\prime},-K+i K^{\prime},-K$, os quais são os vértices deste retângulo, onde

$$
K=K(k)=\int_{0}^{1} \frac{d t}{\sqrt{\left(1-t^{2}\right)\left(1-k^{2} t^{2}\right)}}
$$

é integral elíptica completa de primeira espécie e

$$
K^{\prime}=K^{\prime}(k)=\int_{1}^{1 / k} \frac{d t}{\sqrt{\left(t^{2}-1\right)\left(1-k^{2} t^{2}\right)}}=K\left(k^{\prime}\right), \quad k^{2}+k^{\prime 2}=1 .
$$

A função inversa de (1.2) tem a forma

$$
z=\operatorname{sn}(w, k), \quad 0<k<1
$$

e chama-se seno elíptico $[2,6]$; essa função realiza a transformação conforme do retângulo ao semiplano superior.

A tarefa principal deste trabalho é encontrar o valor de $k, 0<k<1$, que satisfaz o problema considerado.

\section{Transformação Conforme do Quadrado Unitário no Círculo Unitário}

Para iniciarmos a construção do mapeamento conforme do quadrado unitário (Figura 1) para o círculo unitário (Figura 2), sob o qual os vértices do quadrado são levados em quatro pontos eqüidistantes da circunferência unitária: $z_{1}, z_{2}, z_{3}, z_{4}$, introduzimos uma região auxiliar: semiplano superior do plano $\zeta$ (Figura 3). 


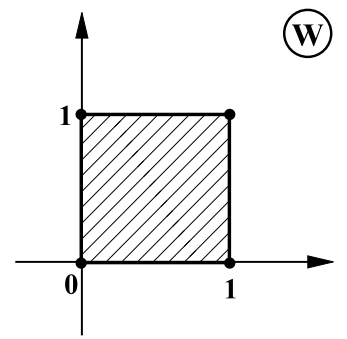

Figura 1: Quadrado unitário.

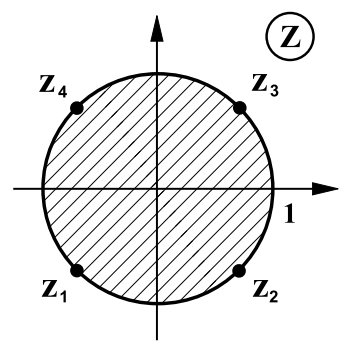

Figura 2: Círculo unitário.

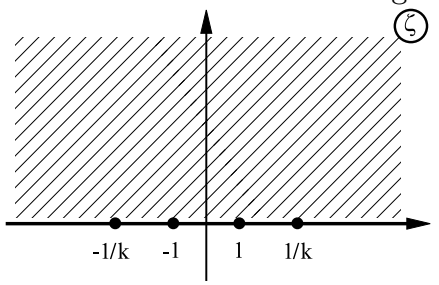

Figura 3: Semiplano superior.

Dividimos este mapeamento em duas partes: transformação do círculo unitário do plano $z$ para o semiplano superior do plano $\zeta$ (chamamos de parte II) e transformação do semiplano superior do plano $\zeta$ no quadrado unitário do plano $w$ (chamamos de parte I); veja Figura 4. Depois, tomaremos a composição das funções inversas destas transformações e obteremos a função que transforma o quadrado unitário do plano $w$ no círculo unitário do plano $z$.

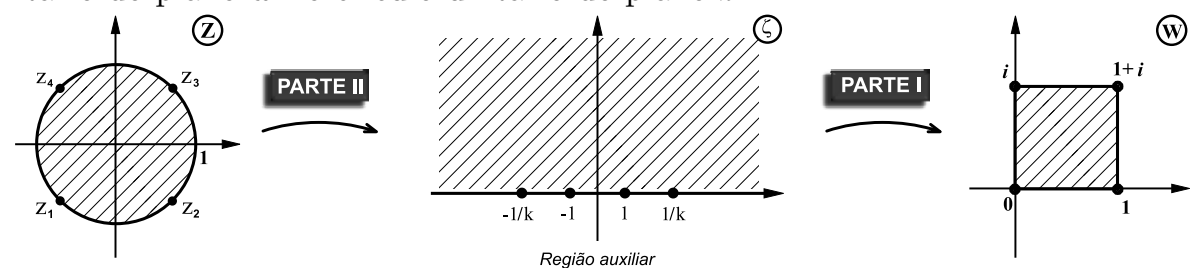

Figura 4: Esquema da resolução.

Agora, construiremos a transformação da parte I, ou seja, transformação conforme do semiplano superior do plano $\zeta$ no quadrado unitário do plano $w$, de tal modo que os pontos do eixo real $-1 / k,-1,1,1 / k$ passam, respectivamente, nos pontos $i, 0,1,1+i$, que são os vértices deste quadrado.

Sabemos que a função $w_{1}=F(\zeta, k)$, onde $F(\zeta, k)$ é definida pela fórmula (1.2), transforma o semiplano superior num retângulo, tal que os pontos do eixo real 1 , $1 / k,-1 / k,-1$ (veja Figura 3) passam, respectivamente, nos pontos $K, K+i K^{\prime}$, $-K+i K^{\prime},-K$, os quais são os vértices deste retângulo (veja Figura 5). Mas, nosso problema é encontrar o valor do módulo $k$, tal que a imagem (retângulo do plano $w_{1}$ ) da transformação de Schwarz-Christoffel seja um quadrado, ou seja, $2 K=K^{\prime}$. Para iniciarmos a resolução deste problema consideramos uma região intermediária, isto é, consideramos no plano $w_{2}$ um retângulo com vértices nos pontos $\pm K_{1}, \pm K_{1}+i K_{1}^{\prime}$ para o qual $K_{1}=K_{1}^{\prime}$ (Figura 6 ). Neste caso da igualdade $K_{1}\left(k_{1}\right)=K_{1}^{\prime}\left(k_{1}\right)=K_{1}\left(k_{1}^{\prime}\right)$ temos que $k_{1}=k_{1}^{\prime}$ e da condição $k_{1}^{2}+k_{1}^{\prime 2}=1$ segue que $k_{1}=k_{1}^{\prime}=\frac{\sqrt{2}}{2}$. 


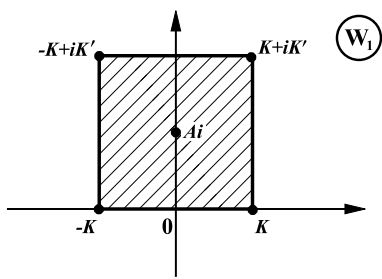

Figura 5: Retângulo.

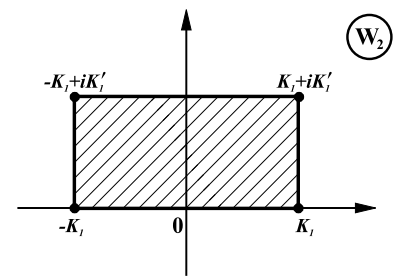

Figura 6: Retângulo $\operatorname{com} K_{1}^{\prime}=K_{1}$.

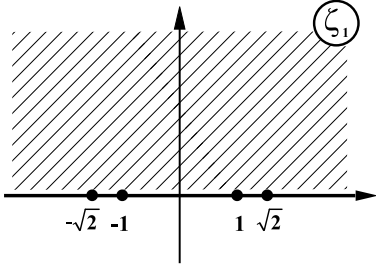

Figura 7: Semiplano superior $\operatorname{com} k_{1}=\frac{\sqrt{2}}{2}$.

Logo a transformação de Schwarz-Christoffel,

$$
w_{2}=F\left(\zeta_{1}, k_{1}\right)=\int_{0}^{\zeta_{1}} \frac{d \tau}{\sqrt{\left(1-\tau^{2}\right)\left(1-k_{1}^{2} \tau^{2}\right)}}, \quad k_{1}=\frac{\sqrt{2}}{2},
$$

transforma biunivocamente o semiplano superior do plano $\zeta_{1}$ (Figura 7 ) no retângulo do plano $w_{2}$, para o qual temos $K_{1}=K_{1}^{\prime}$ (Figura 6 ). Notamos que com esta transformação o intervalo $[-1,1]$ do eixo real do plano $\zeta_{1}$ passa no intervalo $\left[-K_{1}, K_{1}\right]$ do plano $w_{2}$. Por isso, podemos usar o princípio de simetria de Riemann-Schwarz para prolongar analíticamente as regiões consideradas em relação aos intervalos mencionados. Assim, a função (2.1) transforma biunivocamente todo plano com cortes ao longo dos intervalos $(-\infty,-1]$ e $[1,+\infty)$ do eixo real do plano $\zeta_{1}$ (Figura 8) no retângulo do plano $w_{2}$ com vértices $\pm K_{1}+i K_{1}^{\prime}, \pm K_{1}-i K_{1}^{\prime}$ (Figura 9), ou seja, num quadrado $\left(K_{1}=K_{1}^{\prime}\right)$.

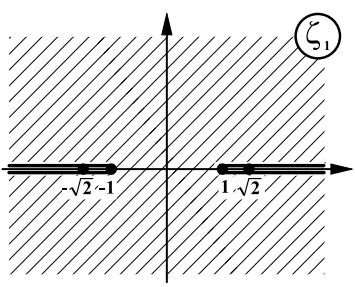

Figura 8: Plano com cortes $(-\infty,-1]$ e $[1,+\infty)$.

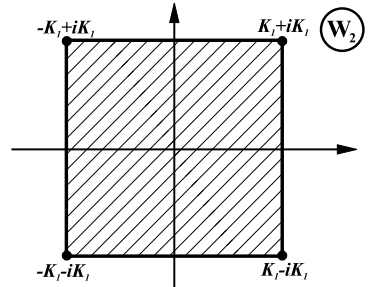

Figura 9: Quadrado com lateral $2 K_{1}$.

Observamos que esta transformação estabelece a seguinte correspondência entre os vértices do quadrado do plano $w_{2}$ e os pontos indicados no semiplano superior do plano $\zeta_{1}$ : o ponto $1 / k_{1}=\sqrt{2}$ que fica na margem superior do corte $[1,+\infty)$ passa no vértice $K_{1}+i K_{1}$; o ponto $1 / k_{1}=\sqrt{2}$ da margem inferior deste corte passa em $K_{1}-i K_{1}$; o ponto $-1 / k_{1}=-\sqrt{2}$ da margem superior do corte $(-\infty,-1]$ é levado em $-K_{1}+i K_{1}$; já o ponto $-1 / k_{1}=-\sqrt{2}$ da margem inferior do último corte citado passa em $-K_{1}-i K_{1}$.

Agora, vamos transformar estas regiões prolongadas dos planos $\zeta_{1}$ e $w_{2}$. Começamos transformando a região do plano $\zeta_{1}$ (Figura 8). Antes de tudo, utilizamos a função $\zeta_{2}=\frac{\zeta_{1}-1}{\zeta_{1}+1}$ e obtemos todo plano com corte ao longo do segmento $[0,+\infty)$ do eixo real no plano $\zeta_{2}$ (Figura 10); com isso os pontos $-\sqrt{2}, \sqrt{2}$ do plano $\zeta_{1}$ passam nos pontos $(\sqrt{2}+1)^{2},(\sqrt{2}-1)^{2}$, respectivamente. Depois, usamos a função $\zeta_{3}=\sqrt{\zeta_{2}}$ (escolhemos um ramo regular desta função plurívoca), a imagem desta 
transformação é o semiplano superior com pontos fixos do eixo real $\pm(\sqrt{2}-1)$, $\pm(\sqrt{2}+1)$ (Figura 11).

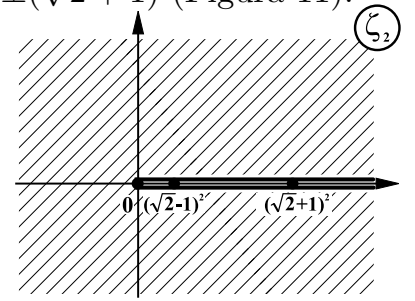

Figura 10: Plano com corte $[0,+\infty)$.

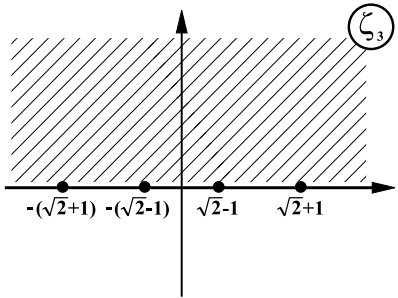

Figura 11: Semiplano superior.

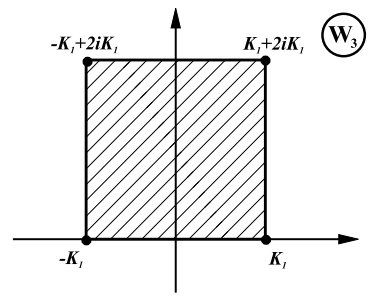

Figura 12: Quadrado com lateral $2 K_{1}$.

Para voltarmos ao semiplano superior da Figura 3 é suficiente fazer extensão do plano $\zeta_{3}: \zeta=(\sqrt{2}+1) \zeta_{3} ;$ com esta transformação os pontos $\pm(\sqrt{2}-1), \pm(\sqrt{2}+1)$ do plano $\zeta_{3}$ passam nos pontos fixos $\pm 1, \pm 1 / k$ do plano $\zeta$, com $k=(\sqrt{2}-1)^{2}$.

A cadeia das transformações construídas relaciona da seguinte maneira os pontos fixos do plano $\zeta$ (Figura 3 ) e os vértices do quadrado do plano $w_{2}$ (Figura 9): o ponto 1 é levado no vértice $K_{1}+i K_{1}$ (lembrando que $\left.K_{1}=K_{1}^{\prime}\right)$; o ponto $1 / k=(\sqrt{2}+1)^{2}$ em $-K_{1}+i K_{1} ;-1 / k=-(\sqrt{2}+1)^{2}$ em $-K_{1}-i K_{1}$ e -1 em $K_{1}-i K_{1}$.

Iremos transformar, um pouco, o quadrado do plano $w_{2}$. Fazemos rotação pelo ângulo $-\pi / 2$ (para voltarmos a correspondência estabelecida pela função (1.2) entre os pontos da fronteira do semiplano superior e os vértices do retângulo) e translação paralela: $w_{3}=-i w_{2}+i K_{1}$. O resultado desta transformação é o quadrado (Figura 12 ), cuja correspondência dos seus vértices com os pontos fixos do plano $\zeta$ (Figura 3) é a mesma que já foi mencionada para a função (1.2).

Desta forma, temos que a composição de funções construída até aqui, $w_{3}=g(\zeta)$, transforma biunivocamente o semiplano superior do plano $\zeta$ num quadrado do plano $w_{3}$, tal que os pontos fixos $\pm 1, \pm 1 / k$ do plano $\zeta$, onde $k=(\sqrt{2}-1)^{2}$, passam nos vértices de um quadrado do plano $w_{3}$. Ao mesmo tempo, lembramos que a função $w_{1}=F(\zeta, k)$ transforma o semiplano superior num retângulo. Como a função que realiza transformação conforme é única com precisão de uma função homográfica, chegamos a conclusão que a função $w_{1}=F(\zeta, k)$, com módulo $k=(\sqrt{2}-1)^{2}$, transforma biunivocamente o semiplano superior num quadrado com lateral $2 K$, ou seja, encontramos o valor do módulo $k$ para o qual teremos, através da integral de Schwarz-Christoffel, a transformação desejada. O valor de $K=K(k)$ é calculado pela fórmula (1.3) ou pode ser encontrado em tabelas [4], usando o valor do módulo $k=(\sqrt{2}-1)^{2}$. E, finalmente, com a função linear $w=\frac{1}{2 K}\left(w_{1}+K\right)$ obtemos o quadrado unitário no plano $w$ (Figura 1).

Assim, a função

$$
w=\frac{1}{2 K} \int_{0}^{\zeta} \frac{d t}{\sqrt{\left(1-t^{2}\right)\left(1-k^{2} t^{2}\right)}}+\frac{1}{2}
$$

transforma o semiplano superior (Figura 3) no quadrado unitário (Figura 1), de tal modo que os pontos da fronteira $-1 / k,-1,1,1 / k$ passam nos vértices deste quadrado $i, 0,1,1+i$, respectivamente. A função inversa de (2.2) tem a forma

$$
\zeta=\operatorname{sn}(2 K w-K, \quad k), \quad K=K(k), \quad k=(\sqrt{2}-1)^{2}
$$


e realiza a transformação inversa.

Iremos construir, agora, a transformação da parte II, isto é, a transformação conforme do círculo unitário do plano $z$ (Figura 2) no semiplano do plano $\zeta$ (Figura 3). Primeiramente, utilizamos a função homográfica

$$
\zeta_{4}=-i \frac{z-1}{z+1}
$$

que transforma o círculo unitário do plano $z$ no semiplano superior. A transformação (2.4) leva qualquer ponto da circunferência unitária, $z=e^{i \theta}$, num ponto do eixo real,

$$
\zeta_{4}\left(e^{i \theta}\right)=-i \frac{e^{i \theta}-1}{e^{i \theta}+1}=\operatorname{tg} \frac{\theta}{2} .
$$

Como, pela condição, temos quatro pontos eqüidistantes na circunferência, então usando rotação do círculo sempre podemos obter o caso quando $z_{1}=e^{-\frac{3 \pi}{4} i}$, $z_{2}=e^{-\frac{\pi}{4} i}, z_{3}=e^{\frac{\pi}{4} i}, z_{4}=e^{\frac{3 \pi}{4} i}$ (como na Figura 2), cujos pontos correspondentes no plano $\zeta_{4}$ são: $\zeta_{4}^{(1)}=-\operatorname{tg} \frac{3 \pi}{8}=-\sqrt{\frac{1-\cos (3 \pi / 4)}{1+\cos (3 \pi / 4)}}=-(\sqrt{2}+1), \quad \zeta_{4}^{(2)}=-\operatorname{tg} \frac{\pi}{8}=-(\sqrt{2}-1)$, $\zeta_{4}^{(3)}=\operatorname{tg} \frac{\pi}{8}=\sqrt{2}-1, \zeta_{4}^{(4)}=\operatorname{tg} \frac{3 \pi}{8}=\sqrt{2}+1$.

Fazendo a extensão $\zeta=(\sqrt{2}+1) \zeta_{4}$, chegamos ao semiplano superior no plano $\zeta$ (Figura 3), de tal modo que os pontos indicados no plano $\zeta_{4}$ passam nos pontos fixos $\pm 1, \pm 1 / k$, onde $k=(\sqrt{2}-1)^{2}$. Desta forma, obtemos o mapeamento da parte II, ou seja, a função

$$
\zeta=-i(\sqrt{2}+1) \frac{z-1}{z+1}
$$

transforma biunivocamente o círculo unitário do plano $z$ no semiplano superior do plano $\zeta$, tal que os pontos eqüidistantes da círcunferência unitária $e^{ \pm \frac{\pi}{4}} i, e^{ \pm \frac{3 \pi}{4}} i$ são levados nos pontos $\pm 1, \pm(\sqrt{2}+1)^{2}$. A função inversa de (2.5) tem a forma

$$
z=\frac{i(\sqrt{2}+1)-\zeta}{i(\sqrt{2}+1)+\zeta}
$$

e transforma biunivocamente o semiplano superior do plano $\zeta$ (Figura 3) no círculo unitário (Figura 2) com a correspondência indicada dos pontos da fronteira. Como já mostramos, a função (2.3) leva o quadrado unitário (Figura 1) no semiplano superior de tal modo que os vértices do quadrado passam nos pontos \pm 1 , $\pm 1 / k= \pm(\sqrt{2}+1)^{2}$ do eixo real no plano $\zeta$ (Figura 3). Então, a composição das funções (2.3) e (2.6), isto é, a função

$$
z=\frac{i(\sqrt{2}+1)-\operatorname{sn}(2 K w-K, k)}{i(\sqrt{2}+1)+\operatorname{sn}(2 K w-K, k)}, \quad K=K(k), \quad k=(\sqrt{2}-1)^{2}
$$

transforma biunivocamente o quadrado unitário no círculo unitário, de tal modo que os vértices do quadrado unitário passam em quatro pontos eqüidistantes da circunferência unitária. 


\section{Algumas Observações}

Observação 3.1. Notamos que usando os mesmos raciocínios como na construção da transformação conforme do semiplano superior no quadrado, podemos calcular o valor exato do módulo $k$ para transformação conforme do semiplano superior num retângulo com razão dos lados $2^{p}$, onde $p$ é qualquer número inteiro.

Observação 3.2. Provaremos que a transformação construída leva o centro do quadrado no centro do círculo. Como no problema analisado neste trabalho, consideramos a situação inversa. O centro do círculo fica no ponto $z_{0}=0$. Com a transformação $(2.5)$ o ponto $z_{0}=0$ passa no ponto $\zeta_{0}=(\sqrt{2}+1) i$. Notamos que a transformação (1.2) leva os pontos do semieixo imaginário superior $[0, i \infty]$ nos pontos do intervalo do eixo imaginário $\left[0, i K^{\prime}\right]$ do plano $w_{1}$. Então, o ponto $\zeta_{0}=(\sqrt{2}+1) i$ passa num ponto $A i \in\left[0, i K^{\prime}\right], A>0$, onde

$$
A i=\int_{0}^{i(\sqrt{2}+1)} \frac{d t}{\sqrt{\left(1-t^{2}\right)\left(1-k^{2} t^{2}\right)}} .
$$

Fazendo a mudança de variável $t=i \tau$ na integral (3.1), temos

$$
A=\int_{0}^{\sqrt{2}+1} \frac{d \tau}{\sqrt{\left(1+\tau^{2}\right)\left(1+k^{2} \tau^{2}\right)}} .
$$

Agora, calculamos a diferença entre os números $i K^{\prime}$ e $A i$ (veja Figura 5):

$$
i K^{\prime}-i A=\int_{0}^{+i \infty} \frac{d t}{\sqrt{\left(1-t^{2}\right)\left(1-k^{2} t^{2}\right)}}-\int_{0}^{i(\sqrt{2}+1)} \frac{d t}{\sqrt{\left(1-t^{2}\right)\left(1-k^{2} t^{2}\right)}} .
$$

Usando na última expressão a mesma mudança de variável, $t=i \tau$, utilizada em (3.1), obtemos

$$
K^{\prime}-A=\int_{\sqrt{2}+1}^{+\infty} \frac{d \tau}{\sqrt{\left(1+\tau^{2}\right)\left(1+k^{2} \tau^{2}\right)}} .
$$

Fazendo na integral acima mais uma troca de variável: $\tau=\frac{1}{k \theta}$, e lembrando que $k=(\sqrt{2}-1)^{2}$, obtemos

$$
K^{\prime}-A=\int_{0}^{\sqrt{2}+1} \frac{d \theta}{\sqrt{\left(1+\theta^{2}\right)\left(1+k^{2} \theta^{2}\right)}} .
$$

Comparando (3.2) e (3.3) vemos que $K^{\prime}-A=A$, isto é, o ponto $A i$ fica exatamente no centro do quadrado do plano $w_{1}$. Como a função linear $w=\frac{1}{2 K}\left(w_{1}+K\right)$ não muda a disposição dos pontos, então $A i$ passa no centro do quadrado unitário.

Observação 3.3. Voltamos à integral de Schwarz-Christoffel (1.2). Como já foi dito essa integral transforma de modo biunívoco o semiplano superior num retângulo e com isso os pontos simétricos do eixo real $\pm 1, \pm 1 / k$ passam nos vértices deste 
retângulo. Agora, consideraremos o caso, quando temos o semiplano superior com quatro pontos fixos quaisquer do eixo real, que têm que passar nos vértices do retângulo e mostraremos que este caso pode ser reduzido ao já analisado com pontos simétricos do eixo real.

Realmente, sejam $x_{1}, x_{2}, x_{3}, x_{4}$ pontos fixos quaisquer do eixo real: $x_{1}<x_{2}<x_{3}<x_{4}$. Construímos duas circunferências: $\Gamma_{1}$ com diâmetro $\left[x_{1}, x_{2}\right]$ e $\Gamma_{2}$ com diâmetro $\left[x_{3}, x_{4}\right]$ (veja Figura 13).

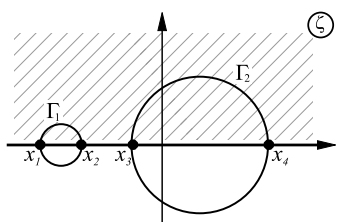

Figura 13: Construção das circunferências $\Gamma_{1}$ e $\Gamma_{2}$.

A circunferência $\Gamma_{1}$ tem centro no ponto $a_{1}=\frac{x_{2}+x_{1}}{2}$ e raio $r_{1}=\frac{x_{2}-x_{1}}{2}, \Gamma_{2}$ tem centro $a_{2}=\frac{x_{4}+x_{3}}{2}$ e raio $r_{2}=\frac{x_{4}-x_{3}}{2}$. Encontramos pontos simétricos simultaneamente em relação a $\Gamma_{1}$ e $\Gamma_{2}$. Denotamos estes pontos por $\zeta^{\prime}$ e $\zeta^{\prime \prime}$ e notamos, pelo sentido geométrico dos pontos simétricos $\zeta^{\prime}$ e $\zeta^{\prime \prime}$, que eles são números reais. Então, $\zeta^{\prime}$ e $\zeta^{\prime \prime}$ satisfazem ao seguinte sistema

$$
\left\{\begin{array}{l}
\left(\zeta^{\prime}-a_{1}\right)\left(\zeta^{\prime \prime}-a_{1}\right)=r_{1}^{2} \\
\left(\zeta^{\prime}-a_{2}\right)\left(\zeta^{\prime \prime}-a_{2}\right)=r_{2}^{2}
\end{array} .\right.
$$

Resolvemos o sistema (3.4) e obtemos os pontos $\zeta^{\prime}$ e $\zeta^{\prime \prime}$.

Consideramos a função homográfica

$$
\omega=\frac{\zeta-\zeta^{\prime}}{\zeta-\zeta^{\prime \prime}}
$$

Sabemos que funções homográficas levam qualquer circunferência numa circunferência $[2,6]$, isto é, as circunferências $\Gamma_{1}$ e $\Gamma_{2}$ passam nas circunferências $C_{1}$ e $C_{2}$ do plano $\omega$. Além disso, as funções homográficas possuem a propriedade de invariância dos pontos simétricos [6,7], de onde segue que os pontos $\zeta^{\prime}$ e $\zeta^{\prime \prime}$, simétricos em relação a $\Gamma_{1}$ e $\Gamma_{2}$ no plano $\zeta$, passam pela transformação (3.5) nos pontos 0 e $\infty$ do plano $\omega$, que são simétricos simultaneamente a $C_{1}$ e $C_{2}$. Isso significa que $C_{1}$ e $C_{2}$ são circunferências concêntricas com centro no ponto 0 . Então, os pontos $x_{1}$, $x_{2}, x_{3}, x_{4}$ passam em pontos simétricos em relação a origem das coordenadas do plano $\omega$. Cabe salientar que a função (3.5) transforma biunivocamente o semiplano superior do plano $\zeta$ no semiplano superior ou inferior do plano $\omega$. Usando agora a expansão ou contração e, se for preciso, a rotação, chegamos à situação da Figura 3 , já analisada.

Observação 3.4. Utilizando os mesmos raciocínios da observação anterior, podemos provar a seguinte afirmação: se é dada uma circunferência com quatro pontos fixos quaisquer, então usando a transformação homográfica, é possível transformar a circunferência dada em outra (com centro na origem) de tal modo que quatro pontos fixos dados passam nos pontos simétricos em pares em relação aos 
eixos das coordenadas.

\title{
Agradecimentos
}

Agradecemos a agência de pesquisas $\mathrm{CNPq}$ que apoiou essa pesquisa com a bolsa 300057/2004-0.

\begin{abstract}
Conformal transformation of the unit square onto the unit circle, which maps square vertices on four equidistant circle boundary points is considered. The construction of this mapping is made by applying the properties of elementary complex value functions, the main principles of conformal mappings, the symmetry principle of Riemann-Schwarz and, in particular, the Schwarz-Christoffel integral.
\end{abstract}

\section{Referências}

[1] A. Bourchtein, L. Bourchtein, B. Herreira, Algumas questões da geração de grades ortogonais computacionais, Mecânica Computacional, 22 (2003), 22822294.

[2] C. Carathéodory, "Conformal Representation", Dover Pub., New York, 1998.

[3] G.M. Goluzin, "Geometric theory of functions of a complex variable", Mathematical monographs, Amer. Math. Soc., Vol. 26, 1969.

[4] E. Jahnke, F. Emde e F. Lösch, "Tables of Higher Functions", McGraw-Hill, New York, 1960.

[5] P. Knupp e S. Steinberg, "Fundamentals of grid generation", CRC Press, Boca Raton, 1993.

[6] Z. Nehari, "Conformal Mapping", Dover Pub., New York, 1997.

[7] W. Rudin, "Real and Complex Analysis", McGraw-Hill, New York, 1987. 